\title{
Unicystic Ameloblastoma in a 9-year-old Child Treated with a Combination of Conservative Surgery and Orthodontic Treatment: A Case Report
}

\author{
Tingting Guo ${ }^{1}$, Ci Zhang ${ }^{1}$, and Jian Zhou ${ }^{1}$ \\ ${ }^{1}$ Beijing Stomatological Hospital
}

November 7, 2021

\begin{abstract}
a 9-year-old girl with UAM of the mandible, which also involved the impaction of the first and second premolars. Marsupialization with orthodontic treatment was performed to shrink the lesion and upright the first premolar involved in the tumor. Ten months after marsupialization, the lesion was completely healed.
\end{abstract}

\section{Introduction}

Unicystic ameloblastoma (UAM) is defined as a variant of intraosseous ameloblastoma that occurs as a single cystic cavity. UAM is believed to be less aggressive, and it responds more favorably to conservative treatments than the multicystic or solid types ${ }^{1}$. Various treatment modalities such as segmental or marginal resection for UAM have been used, similar to those generally used for conventional ameloblastoma, and more conservative treatments have also been frequently reported ${ }^{2}$. The traditional treatment of complete resection of the lesion site could impact a growing young patient's physical and psychological development. Therefore, conservative treatments are widely applied for treating UAM in children.

This report presents a case of mandibular UAM in a 9-year-old girl who was successfully treated by marsupialization and orthodontic treatment without the need for teeth removal.

\section{Case report}

A 9 years and 2 months year old female with a chief report of swelling in the left mandibular premolar region was referred to our medical center. The patient had no systemic health conditions. Extraoral examination revealed facial symmetry, and intraoral examination revealed mucosal swelling extending from the left lower first deciduous molar to the left second deciduous molar region, mixed dentition, and unerupted left lower first and second premolar. (Figure $1 \mathrm{~B}, \mathrm{C}$ )

A panoramic radiograph revealed a well-circumscribed, unilocular radiolucency in the region of the left lower premolars, extending from the distal canine area to the mesial root of the first molar area, with an unerupted first and second premolar that located lower than the healthy right side. The roots of both left lower first and second deciduous molar were absorbed to the neck of the teeth. (Figure $1 \mathrm{~A}$ )

Cone-beam computed tomography (CBCT) was performed to confirm the location of the lesion and the relationship between the lesions and their adjacent anatomical structures. (Figure 4 A, B, C)

The cystic lesion was treated by marsupialization together with the extraction of the left lower first and second deciduous molar, and biopsy was conducted (age: 9 years and 2 months), which was performed to 
shrink the lesion size. Ibuprofen is used to relieve pain after surgery. After histopathologic evaluation, the lesion was diagnosed as UAM. (Figure 2)

Radiographs obtained five months (age: 9 years and 7months) after marsupialization showed that the lesion margin had lost clarity and that regenerated bone replaced the low-density area. The first and second premolars had begun to erupt. However, the direction of the eruption of the first premolar was mesioangular, and no space was present between the left canine and the first premolar. (Figure $3 \mathrm{~A}, \mathrm{D}$ )

Thus, a segment arch appliance was bonded to the left canine and first molar of the mandible to keep leeway space with coil spring. Then, an orthodontic button was bonded to the left first premolar, and distal traction was applied to the tooth using an elastic power chain. (Figure $3 \mathrm{~B}, \mathrm{E}$ )

Five months after the orthodontic appliance placement (age: 10 years), we observed that the first premolar erupted in place. (Figure $3 \mathrm{C}, \mathrm{F}$ )

CBCT invested ten months (age: 10 years) after marsupialization showed that the radiolucent area was disappeared entirely. (Figure 4 D, E, F)

\section{Discussion}

In children, the treatment of unicystic ameloblastoma is complicated by the key factors: Continuing facial growth, increased bone turnover rate, and presence of unerupted teeth. There is a consensus in various literature that ameloblastoma has to be treated aggressively to avoid recurrences, but there is a dilemma on the applicability of an initial radical, extensive surgery in children.

Given that the mandible resection in pediatric patients may lead to complications, such as dysfunction and deformity, our patient was submitted to conservative treatment considering preserving as much bone as possible to avoid aesthetic deformities.

Marsupialization is considered for the exteriorization or decompression of the cyst. Marsupialization followed by enucleation is one therapeutic approach for ameloblastoma ${ }^{3}$. which is our original treatment plan. Surprisedly, after 10 months, we observed that the lesion was completely healed. There are several reports of cystic ameloblastoma cases in which the tumor completely disappeared after marsupialization alone, suggesting that marsupialization helps avoid wide resection of the mandible in patients with $\mathrm{UAM}^{4}$. For our case, we assumed the lesion fully recovered because the pathological classification is simply cystic (type 1), which favored successful outcomes with conservative management.

In the present case, the lesion was histologically diagnosed as ameloblastoma at the biopsy, and we assumed that the tumor was not entirely enucleated by marsupialization and remained around the crown of the first and second premolar. Accordingly, we performed careful follow-up to monitor the tumor in size. Orthodontic treatment was applied in addition to marsupialization to allow the teeth to erupt. Similar to our case, orthodontic treatment was successfully applied in some reported cases of tumors involving the crown of the unerupted teeth ${ }^{5}$. After 10 months, the lesion completely disappeared and the first and the second premolar erupted to a normal level. Long-term follow-up with radiography is mandatory for early detection of late recurrence. We are still monitoring our patients annually using radiography.

Conclusively, the treatment avoids a resection of the mandible and induces eruption of the teeth involved in the tumor. Thus, the combination of conservative surgery and orthodontic treatment was influential in managing a UAM among the pediatric population.

\section{Bullet Points}

- Conservative surgery should be the preferred approach for UAM management in children to avoid dysfunction and deformity.

- Multidisciplinary cooperation benefits the patient, as the orthodontic treatment intervention could promote the eruption of the teeth involved in UAM.

- This report documents that marsupialization alone may have the potential to cure UAM. 
Conflict of interest

The authors declare no conflict of interest.

\section{References}

1 Seintou A, Martinelli-Kläy CP, Lombardi T. Unicystic ameloblastoma in children: systematic review of clinicopathological features and treatment outcomes. International Journal of Oral and Maxillofacial Surgery 2014; 43 : 405-412.

2 Kim J, Nam E, Yoon S. Conservative management (marsupialization) of unicystic ameloblastoma: literature review and a case report.Maxillofac Plast Reconstr Surg 2017; 39 : 38.

3 Yang Z, Liang Q, Yang L et al. Marsupialization of mandibular cystic ameloblastoma: Retrospective study of 7 years. Head $\&$ Neck 2018; $40: 2172-2180$.

4 Nakamura N, Higuchi Y, Mitsuyasu T, Sandra F, Ohishi M. Comparison of long-term results between different approaches to ameloblastoma. Oral Surgery, Oral Medicine, Oral Pathology, Oral Radiology, and Endodontology 2002; 93 : 13-20.

5 Erdur EA, Ileri Z, Ugurluoglu C, Cakir M, Dolanmaz D. Eruption of an impacted canine in an adenomatid odontogenic tumor treated with combined orthodontic and surgical therapy. American Journal of Orthodontics and Dentofacial Orthopedics 2016; 149 : 923-927.

\section{Figure legend}

Figure 1.

A, A panoramic radiograph obtained at the first visit revealing a unilocular, well-defined, radiolucent lesion surrounding the unerupted left lower first and second premolar.

B, C Intraoral photographs. Arrow indicates mucosal swelling.

Image obtained at 9 years and 2 months of age.

Figure 2. The histological findings (10x) of the specimen obtained at the marsupialization revealing a simple cystic type (type I) ameloblastoma. Arrow indicates loosely arranged suprabasal cells. Specimen obtained at 9 years and 2 months of age.

Figure 3.

A, D Postoperative intraoral photograph and radiograph were taken after 5 months shows the direction of the eruption of the first premolar was mesioangular, and no space was present between the left canine and the first premolar. The lesion margin had lost clarity, and that regenerated bone replaced the low-density area. Image obtained at 9 years and 7 months of age.

B, E Segment arch appliance was bonded to the left canine and first molar of the mandible to keep leeway space with coil spring, and distal traction was applied to the tooth using an elastic power chain.

C, F Intraoral photograph and radiograph were taken five months after the orthodontic appliance placement, the first premolar erupted in place. Image obtained at 10 years of age.

Figure 4.

A, B, C, CBCT images were taken on the first visit. A. CBCT 3D reconstruction shows a low-density area surrounding the unerupted left lower first and second premolar. B. Axial CBCT view shows an expansion of the buccal and lingual cortices in the mandible. C. Coronal CBCT view at the level of the mandible. Image obtained at 9 years and 2 months of age.

D, E, F, Postoperative CBCT taken after 10 months. D. CBCT 3D reconstruction that regenerated bone surrounding the left lower first and second premolar. E. Axial CBCT view shows normal trabeculae replaced 
the low-density area in the mandible. F. Coronal CBCT view at the level of the mandible. Image obtained at 10 years of age.
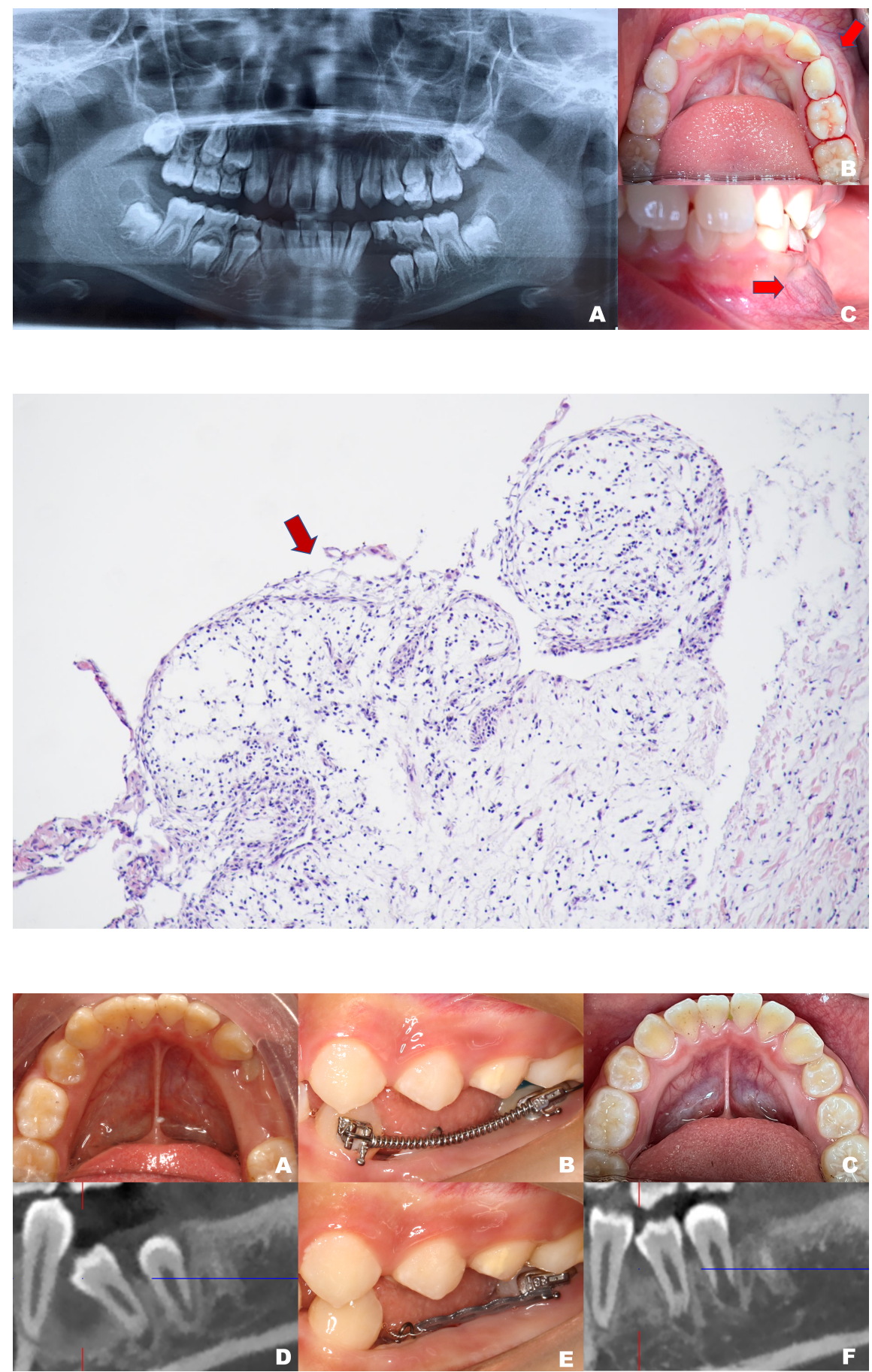


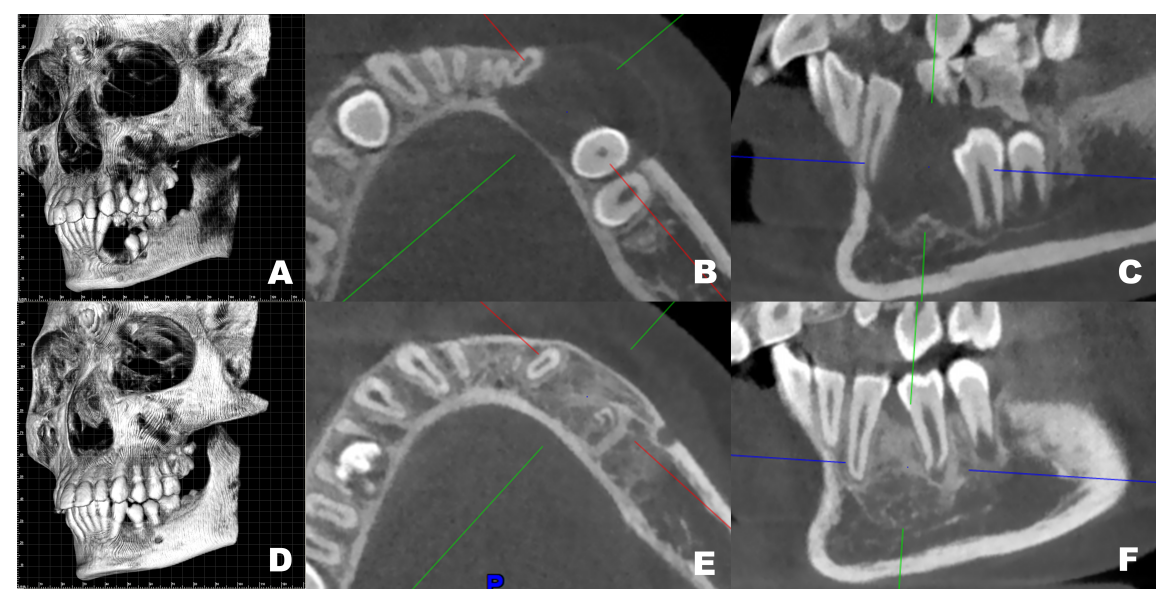

